\title{
Metodología de clasificación de señales electromiográficas
}

\author{
J.R. Caro Vásquez ${ }^{1}$, J.I. Chairez Oria², C. Yáñez Márquez ${ }^{1}$ \\ ${ }^{1}$ Instituto Politécnico Nacional, Centro de Investigación en Computación, \\ México \\ ${ }^{2}$ Instituto Politécnico Nacional, Unidad profesional multidisciplinaria de Biotecnología, \\ México
}

\begin{abstract}
Resumen. In recent years, there has been extensive use electromyographic signals (EMG's) as the primary inputs on algorithms used in control of smart prosthesis; at the same time, the use of classification algorithms to identify patterns in upper limb movements is rising. Some of the main challenges that exist in classification are; EMG's feature extraction and high complexity of algorithms that have been used for that application. This paper present a proposal solution to the problematic mentioned before, a pattern classification method using energy as the only feature and an algorithm capable of running in parallel whose principal function is make the classifying task faster than another conventional algorithm.
\end{abstract}

Palabras clave: electromiografía, EMGs, patrones, características, prótesis, clasificadores dedicados, energía.

\section{Introducción}

La electromiografía superficial ha sido el área de investigación principal para el control de prótesis de miembro superior por más de seis décadas. [1], [2].

Con fin de mejorar de mejorar el control de prótesis mioeléctricas, en las últimas dos décadas se ha investigado las metodologías de extracción de características haciendo uso de electrodos superficiales. Estos esfuerzos han estado enfocados principalmente en el área de reconocimiento de patrones, específicamente atendiendo la tarea de clasificación. En la cual se asume que las EMGs tienen patrones que pueden ser identificados por un algoritmo de cómputo inteligente. [1,3].

Hoy en día, las EMGs son las principales señales utilizadas para el control en prótesis mioeléctricas de miembro superior. En el estado del arte, varios métodos distintos de clasificación de patrones y diferentes características extraídas de la señal se han utilizado con anterioridad. Por citar solo algunos ejemplos, podemos mencionar que Xing et al [4] hacen uso de máquinas de soporte vectorial (SVM) para la clasificación de las EMGs, Yousefi et al [5] mencionan sus aplicaciones con Redes neuronales artificiales (ANN) con el mismo propósito. Por otro lado, la extracción de características de señales cuya función es extraer información de las EMGs es un problema abierto ya que existe un sinfín de posibles técnicas de procesamiento de 
señales que pueden ser usadas para esta aplicación. Este hecho queda evidenciado en los trabajos de Phinyomark et al [6] quienes enfatizan el uso de área bajo la curva de EMGs (IEMG), la media absoluta (MAV), la varianza (VAR), el valor efectivo de la señal (RMS), y doce características más extraídas década una de las señales, en cambio Gokgoz et al [7] prefieren utilizar únicamente la transformada de ondícula (DWT).

Con objeto de mejorar el rendimiento de un algoritmo y tener un menor tiempo de procesamiento, se hacen uso de técnicas de procesamientos, entre ellas destaca principalmente las técnicas de selección de características las principales técnicas utilizadas son análisis de componentes principales (PCA), y análisis de componentes mutuos (MCA) [8].

En el presente trabajo se plantea y describe una metodología de clasificación de patrones, que se enfoca en la extracción de una característica única, para este caso se hace el uso de la energía de la EMGs. Se presenta, además, una solución para modificar algoritmos de clasificación de patrones capaz de realizar cómputos en paralelo, lo cual permite clasificar más rápidamente que algoritmos convencionales.

El resto del documento se encuentra organizado de la siguiente forma: en la Sección 2, se presenta la metodología y las características del experimento, en la Sección 3, se presentan los resultados obtenidos y una pequeña discusión sobre ellos, finalmente en la sección 4 se presentan algunas conclusiones.

\section{Metodología}

En esta sección se presenta de manera detallada la naturaleza de los experimentos y la descripción del banco de datos, se muestra también el pre procesamiento de la EMGs, los modelos de clasificación de señales y la arquitectura del modelo final.

Banco de datos

El banco de datos que se utiliza en la presente investigación lo realizaron los investigadores Rami N. Khusaba, Sarath Kodagoda, Dikai Liu y Gamini Dissanayake miembros del grupo de investigación de la universidad tecnología de Sydney y está disponible para la comunidad científica en (www.rami-khushaba.com/electromyogram-emg-repository.html).

El banco está conformado por señales de EMG obtenidas de todo alrededor del antebrazo por 8 electrodos superficiales (DE 2.x series EMG sensors) y es procesado por (Bagnoli desktop EMG system from Delsys Inc). La señal fue amplificada con una ganancia de 1000,recuperada con un convertidor analógico-digital de 12 bits (National Instruments, BNC-2090) a una frecuencia de muestreo de $4000 \mathrm{~Hz}$, y filtrada con un pasabanda de frecuencias entre $20 \mathrm{~Hz}-450 \mathrm{~Hz}$ y un filtro notch de $50 \mathrm{~Hz}$ para eliminar la frecuencia de la red eléctrica.

Los datos se conforman de pruebas de 5 s. tomadas a 8 personas diferentes , 6 hombres y 2 mujeres con miembros totalmente sanos (sin ningún desorden muscular).

Durante este experimento fueron recolectados 15 diferentes posiciones del miembro i.e., Thumb (T), Index (I), Middle (M), Ring (R), Little (L) los combinados ThumbIndex (T-I), Thumb-Middle (T-M), Thumb-Ring (T-R), Thumb-Little (TL), IndexMiddle (I-M), Middle-Ring (M-R), Ring-Little (RL), Index-Middle-Ring (I-M-R), 
Middle-Ring-Little (M-RL), y finalmente la clase Hand close (HC), se tomó prueba de cada una de ellas 6 veces a cada persona por un periodo de tiempo de 5 . El autor recomienda utilizar 4 de ellas como entrenamiento y 2 de prueba para validación.

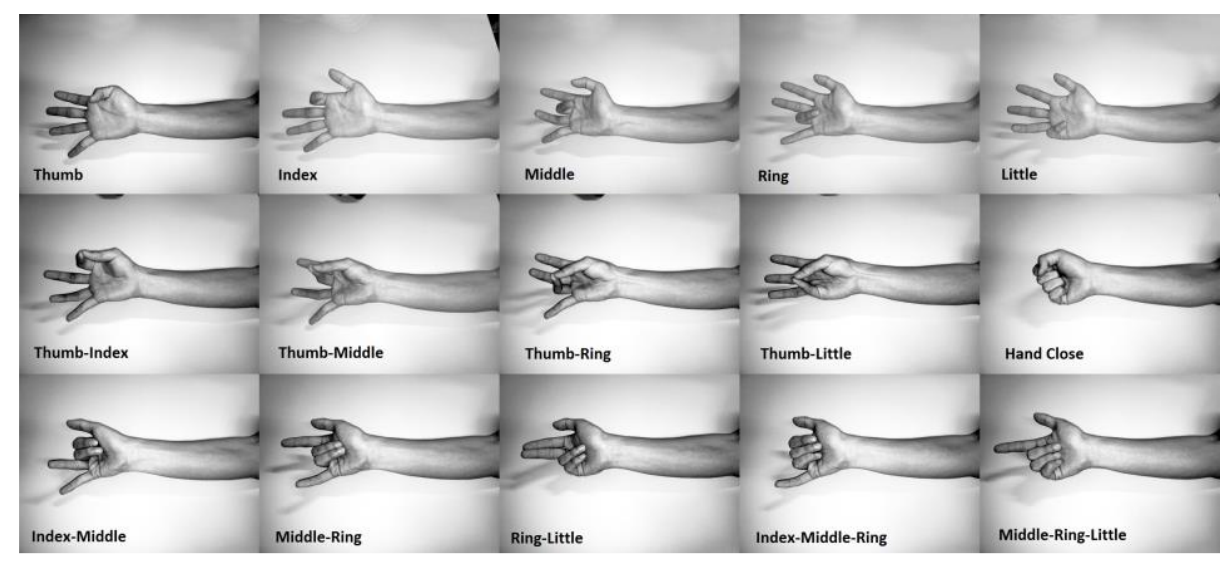

Fig. 1. Movimientos de clasificación.

La figura 1 fue recuperada del artículo original de Khushaba et al donde se introduce el banco de datos [8].

\subsection{Extracción de características}

La metodología propuesta en este artículo consiste en tomar una única característica de la señal, tal que represente información suficiente para representar la señal en un rasgo numérico real permitiéndonos omitir el pre procesamiento de selección de características.

En este caso en particular se toma la única característica de energía de la señal en tiempo discreto por un periodo de tiempo de $0.25 \mathrm{~s}$. generando un nuevo banco de datos cuyos patrones están conformados de la siguiente forma [9]:

$$
x^{i}=\sum_{1000 i}^{1000 i+1000} n^{2}
$$

Siendo n la muestra que de señal recuperada por el convertidor analógico- digital.

\subsection{Clasificación}

Se hace uso del software "weka" como apoyo a la clasificación con uso de los modelos más utilizados en el estado del arte [10]. Con ayuda del software se clasifica el banco de datos completo para una sola persona con características de energía para los clasificadores ANN [11, 12, 13, 14]. K-nearest neighbor (KNN) [11, 15]. Clasificadores bayesianos [11, 16]. SVM [17], C4.5 [11]. 
A partir de este punto el experimento se enfoca principalmente en el clasificador que presente un mayor rendimiento.

\subsection{Algoritmo paralelo}

Una vez que conocemos el algoritmo de mayor rendimiento se hace uso de técnicas de ensamble de clasificadores para hacer trabajar un algoritmo de clasificación en forma paralela [18]. En este caso se usarán específicamente los clasificadores dedicados o "one-class clasifier ensemble" [19], cuya función es generar un clasificador en paralelo agregando tantos clasificadores como clases haya y dedicando a cada uno de ellos a enfocarse en los patrones de su clase correspondiente.

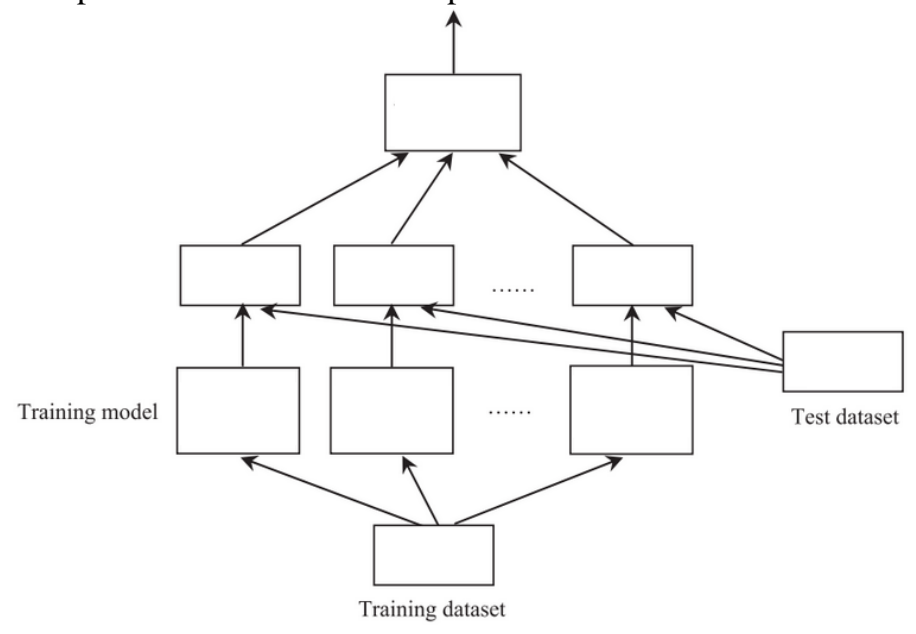

Fig. 2. Esquema de clasificadores dedicados.

La figura anterior muestra el esquema de clasificadores dedicados aplicado a SVM utilizado por Xiang-Yang Wang et al en su trabajo [19].

\section{Resultados}

Una vez generado el banco de datos de energía con las características mencionadas anteriormente tal y como se ha mencionado en el capítulo anterior, el primer experimento del método hace uso del software "weka" para encontrar los resultados de los clasificadores más usados en el estado del arte.

Se prueba el algoritmo con el método Hold-Out tal y como lo recomienda el autor del banco de datos [8]; y además se utiliza el método de validación Leave-One-Out para validar estos modelos de clasificación. Los resultados se muestran en la tabla 1.

Se hace notorio en la tabla anterior que el algoritmo más competitivo para esta aplicación es el KNN. Sin importar que los algoritmos naive bayes y C4.5 hayan mostrado un rendimiento muy bueno con el método de validación leave one out, éstos muestran 
un rendimiento muy pequeño para el caso de hold out; en cambio, el KNN muestra un rendimiento adecuado tanto en Hold-Out y Leave-One-Out.

Tabla 1. Resultados de clasificación en modelos del estado del arte.

\begin{tabular}{ccc}
\hline Clasificador & Hold-Out & Leave-One-Out \\
\hline KNN & .88 & .88 \\
Naive Bayes. & .80 & .80 \\
SVM & .81 & NA \\
C4.5 & .72 & .94 \\
ANN & .81 & NA \\
\hline
\end{tabular}

Para este caso en particular, las máquinas de soporte vectorial y las redes neuronales pueden mejorar su desempeño con una arquitectura y configuración correcta, pero su tiempo de respuesta suele ser muy alto, por lo que no fueron considerados para el experimento.

Una vez elegido el clasificador base, se genera barrido clase por clase identificando el rendimiento del clasificador para diferentes números de clases, el cual nos indicará cuáles y cuántas clases pueden ser consideradas para control de una prótesis mioeléctrica. A partir de este experimento el método de validación utilizado es HoldOut tomando en cuenta la recomendación del autor del banco de datos. La siguiente figura muestra los resultados de este experimento.

Tabla 2. Rendimiento del algoritmo para $\mathrm{n}$ clases.

\begin{tabular}{cccccc}
\hline $\begin{array}{c}\text { Número de } \\
\text { clases }\end{array}$ & rendimiento & $\begin{array}{c}\text { Número de } \\
\text { clases }\end{array}$ & rendimiento & $\begin{array}{c}\text { Número de } \\
\text { clases }\end{array}$ & rendimiento \\
\hline 1 & 1 & 6 & .97 & 11 & .88 \\
2 & 1 & 7 & .92 & 12 & .88 \\
3 & .98 & 8 & .89 & 13 & .87 \\
4 & .98 & 9 & .89 & 14 & .84 \\
5 & .98 & 10 & .88 & 15 & .84 \\
\hline
\end{tabular}

El resultado del experimento anterior nos indica que sin problema podríamos generar un código para el control de una prótesis mioélectrica y su eficiencia dependerá directamente de su rendimiento, queda claro como para 2 clases distintas no existe discriminación alguna.

Una vez hechas estas pruebas, se genera un algoritmo paralelo con el esquema de clasificadores dedicados mostrado en la figura 2 y se evalúa buscando al menos un rendimiento similar al clasificador original para al menos 2 personas distintas como base para evaluar el algoritmo, ya que en cada uno de los casos el algoritmo se comporta de manera diferente. Los resultados del experimento anterior se muestran en la siguiente tabla.

Tabla 3. Resultados de algoritmo KNN dedicado.

\begin{tabular}{ccc}
\hline Persona & KNN & KNN dedicado \\
\hline 1 & 84.33 & 84.33 \\
2 & 79.00 & 79.00 \\
3 & 79.67 & 79.67 \\
4 & 79.00 & 79.00 \\
\hline
\end{tabular}


Aparentemente, el algoritmo dedicado de clasificación no muestra ninguna ventaja; sin embargo, su mayor aportación se encuentra en el tiempo de ejecución, ya que si el proceso se realiza en paralelo, el tiempo de respuesta tanto como para su fase de entrenamiento como su fase de prueba se verá reducido de la siguiente manera.

$$
t_{d}=\frac{t_{c}}{N}
$$

siendo $t_{c}$ el tiempo de respuesta del algoritmo original y $\mathrm{N}$ el número de clasificadores dedicados.

\section{Conclusión}

En este artículo se introduce una metodología rápida para clasificación de EMG con optimización de recursos adecuados para generar algoritmos de control, que pueden ser usados en la implementación de un circuito haciendo uso del concepto de clasificadores dedicados totalmente aplicable en cómputo paralelo y FPGAs.

Se introduce el concepto de energía como característica única de la señal de EMG, y se verifica que este único rasgo proporciona información suficiente para aplicaciones concretas para la clasificación, sin necesidad de un pre-procesamiento.

\section{Referencias}

1. Jiang, N., Vujaklija, I., Rehbaum, H., Graimann, B., Farina, D.: Is Accurate Mapping of EMG Signals on Kinematics Needed for Precise Online Myoelectric Control? IEEE Transactions on Neural Systems and Rehabilitation Engineering, 22, 549-558 (May 2014)

2. Englehart, K., Hugdins, B., Parker, P.: Multifunction Control of Prostheses Using the Myoelectric Signal. Boca Raton, FL: CRC Press (2000)

3. E. Scheme and K. Englehart.: Electromyogram pattern recognition for control of powered upper-limb prostheses: State of the art and challenges for clinical use. J. Rehabil. Res. Develop. 48, 643 (2011)

4. Kexin Xing, Peipei Yang, Jian Huang, Yongji Wang, Quanmin Zhu.: A real-time EMG patter recognition method for virtual myoelectric hand control. Neurocomputing, 136, 345-355 (January 2014)

5. Yousefi, J., Hamilton-wright, A.: Charazterizing EMG data using machinelearning tools. Computers in Biology and Medicine, 51, 1-13 (April 2014)

6. Phinyomark, A., Quaine, F., Charbonnier, S., Serviere, C., Tarpin-Bernard, F., Laurillau, Y.: Feature extraction of the first difference of EMG time series for EMG pattern recognition. Computer Methods and Programs in Biomedicine, 117, 247256 (November 2014)

7. Gokgoz, E., Subasi, A.: Comparison of decision tree algorithms for EMG signal classification using DWT. Biomedical Signal Processing and Control, 18, 138-144 (April 2015) 
8. Khushaba, R.N., Kodagoda, S.: Electromyogram (EMG) Feature Reduction Using Mutual Components Analysis for Multifunction Prosthetic Fingers Control. ICARCV 2012 (December 2012)

9. Oppenheim, A.V., Schaffer, R.W., Jhon R.: BuckDiscrete-Time Signal Processing. Upper Saddle River, New Jersey: Prentice-Hall, Inc. (1999)

10. Witten, I.H., Frank, E., Hall, M.A.: Data Mining Practical Machine Learning Tools and Techniques. Burlington, MA 01803, USA: Elsevier Inc. (2011)

11. Duda, R.O., Hart, P.E., Stork, D.G.: Pattern classification. Wiley, New York (2001)

12. McCulloch, W., Pitts, W.: A logical calculus of the ideas immanent in nervous activity. Bulletin of Mathematical Biophysics, 5, 115-133 (1943)

13. Rosenblatt, F.: The Perceptron: A probabilistic model for information storage and organization in the brain. Psychological Review, 65, 386-408 (1958)

14. Rumelhart, D.E., Hinton, G.E., Williams, R.J.: Learning internal representation by Backpropagating errors. Nature, 323, 533-536 (1986)

15. Cover, T.M., Hart, P.E.: Nearest Pattern Classification. IEEE Trans. on Information Theory, 13, 21-27 (1967)

16. Díaz-de-León, J.L., Yánez-Márquez, G., Sánchez-Garfias, F.A.: Reconocimiento de patrones. Enfoque probabilístico-estadístico. IT 83, Serie Verde, Centro de Investigación en Computación, IPN, México (2003)

17. Cortes, C., Vapnik, V.: Support Vector Network. Machine Learning, 20, 273-297 (1995)

18. Kuncheva, L.: Combining pattern classifiers. Hoboken, New Jersey. John Wiley \& Sons, Inc. (2014)

19. Xiang-Yang Wang, Bei-Bei Zhang, Hong-Ying Yang.: Active SVM-based relevance feedback using multiple classifiers ensemble and features reweighting. Engineering Applications of Artificial Intelligence, 26, 368-381 (January 2013) 